USITP-00-02

OSLO-TP 2-00

March-2000

\title{
Classical phase space and statistical mechanics of identical particles
}

\author{
T.H. Hansson ${ }^{1}$, S.B. Isakov ${ }^{2}$, J.M. Leinaas ${ }^{3}$, and U. Lindström ${ }^{4}$ \\ 1,4 Department of Physics, University of Stockholm, Box 6730,S-11385 Stockholm, Sweden \\ ${ }^{2,3}$ Department of Physics, University of Oslo, P.O. Box 1048 Blindern, N-0316 Oslo, Norway
}

\begin{abstract}
Starting from the quantum theory of identical particles, we show how to define a classical mechanics that retains information about the quantum statistics. We consider two examples of relevance for the quantum Hall effect: identical particles in the lowest Landau level, and vortices in the Chern-Simons Ginzburg-Landau model. In both cases the resulting classical statistical mechanics is shown to be a nontrivial classical limit of Haldane's exclusion statistics.
\end{abstract}

\footnotetext{
e-mail: ${ }^{1}$ hansson@physto.se, ${ }^{2}$ serguei.isakov@fys.uio.no, ${ }^{3}$ j.m.leinaas@fys.uio.no, ${ }^{4}$ ul@physto.se

2 Present address: NumeriX Corporation, 546 Fifth Avenue, New York, NY 10036, USA

1,4 Supported by the Swedish Natural Science Research Council

${ }^{3}$ Supported by the Norwegian Research Council
} 


\section{Introduction}

Particle statistics is usually considered to be a quantum effect. It is expressed through the symmetry of the wave function of a system of identical particles and does not appear, normally, as an interaction in the Hamiltonian. In two space dimensions it can be represented as a special kind of interaction, but being of the Aharonov-Bohm type, it does not give rise to any force on the particles.

Thus, at the level of classical trajectories of individual particles, there is no difference between identical and non-identical particles. There is however one place in the classical description of particles where their indistinguishability is important, namely in the statistical mechanics. There the trajectories of individual particles no longer matters, but the volume of the available phase space is important for thermodynamical quantities. Indistinguishability is introduced by dividing the phase space volume of $N$ non-identical particles with the factor $N$ !. This reduction is essential to give the correct expression for the entropy and thus to resolve Gibbs' paradox.

The reduction in phase space is readily understood. If the particles are indistinguishable all configurations that can be related by a permutation of the particles correspond to one and the same physical configuration. This single configuration for identical particles is then represented as $N$ ! different configurations in the case of distinguishable particles. The configuration space of indistinguishable point particles is therefore derived from the space of distinguishable particles by an identification of equivalent points.

The identification of points implies that the configuration space of a system of identical particles is not everywhere a smooth manifold, there are singularities corresponding to the situation where two or more particles occupy the same point in space. Such a point is a geometrical singularity, a point of infinite curvature. For the phase space the situation is similar, the identification of points introduce singularities, although in general these singularities do not have the same simple geometrical interpretation as in configuration space.

The quantum description of identical particles can be introduced in terms

of wave functions, or alternatively in terms of path integrals, defined on the configuration space with identifications [1, 2]. The presence of singularities then are important, since it divides the continuous paths into different classes, depending on how they evolve around the singularities. Such classes can be 
associated with different phase factors. There is only one characteristic phase factor for each system of identical particles, corresponding to an exchange of two particles, and this factor identifies the statistics. Viewed in this way, the statistics parameter associated with the particles labels inequivalent quantizations of the classical system. Thus, the statistics parameter appears in the quantization of the system and is not present in the classical description of the particles.

In this paper we will to discuss an alternative approach to the classical description of identical particles. This does not mean that we consider the standard description of point particles referred to above as being in any sense incorrect. However, we would like to stress that starting from the quantum theory there are different possibilities for describing the corresponding classical system, and we would like to examine one where the statistics parameter is present also at the classical level. As discussed in the paper we may view this as a non-standard way of taking the classical limit.

The way we introduce the classical description is to consider, in a general form, a coherent state representation of the quantum system. We assume the coherent states to be determined by a set of particle coordinates, and we further assume the time evolution (in the low energy regime) to a good approximation to be described simply by the motion of these coordinates. There is a manifold defined by the set of possible coordinates and a natural phase space structure inherited from the full quantum description. This phase space is a smooth manifold, even when the particle coordinates coincide, and the reduction corresponding to the factor $1 / N$ ! does not have to be introduced by hand, but appears naturally when calculating the phase space volume.

To clarify this idea, we consider the case of a harmonic oscillator coherent state representation of a system of identical point particles in some detail. We show how the classical description introduced in this way distinguishes between bosons, fermions, and in general anyons [2, 3], and we calculate the available phase space volume for the case of $N$ identical particles in a finite volume. The classical statistics parameter is then identified as the phase space volume occupied by each of the particle present in the system. Viewed in this way the description has the character of a classical analogue of the quantum exclusion statistics introduced some time ago by Haldane [4]. We examine this correspondence in some detail by considering the statistical mechanics of our classical system. 
The description we use is not restricted to systems of point particles. We illustrate this by considering vortex solutions of the Chern-Simons GinzburgLandau theory. The manifold defined by the $N$-vortex configurations has a natural phase space structure, and although this can not be fully determined, the phase space volume can be calculated and the statistics parameter identified. This particle description of vortices is closely related to a description of vortices in the (relativistic) abelian Higgs model previously discussed by Samols, Manton and others [5, 6] although in their case the vortex manifold is identified as a configuration space rather than as a phase space.

\section{Classical phase space from the quantum description}

In this section we consider a general quantum system and a subset of states $\left|\psi_{x}\right\rangle$, which is indexed by a set of coordinates $x=\left\{x_{1}, x_{2}, \ldots, x_{N}\right\}$. These may be the coordinates of a system of (identical) particles or the coordinates of an $N$ soliton configuration, but we do not have to be more specific at this point. We only assume that the wave function evolves smoothly with a change of these coordinates, and that it is symmetric under an interchange of any pair of the $N$ coordinates. We furthermore assume, that in the regime of interest (typically at low energies), the time evolution of the system, to a good approximation, can be described (up to a phase factor) as a time evolution of the coordinates only. This means that it makes sense to consider the restricted (constrained) system where the evolution of the system is projected to the manifold defined by the normalized states $\left|\psi_{x}\right\rangle$. Since the physical states correspond to rays in the Hilbert space, i.e. to state vectors defined up to a complex factor, we consider the classical $N$-particle space $\mathcal{M}$, derived from the quantum description, to be defined by the normalized states $\left|\psi_{x}\right\rangle$ only up to such a phase factor. It is the phase space structure of the space $\mathcal{M}$ which will be of importance for our discussion.

The Schrödinger equation of the quantum system can be derived from the Lagrangian,

$$
L=i \hbar\langle\psi \mid \dot{\psi}\rangle-\langle\psi|H| \psi\rangle
$$

where $H$ is the Hamiltonian of the system, and the Lagrangian of the constrained system is obtained from this by restricting $|\psi\rangle$ to the subset of states $\left|\psi_{x}\right\rangle$. Expressed in terms of the coordinates $x$, it has the generic form

$$
L=\dot{x}_{i} A_{i}(x)-V(x),
$$


where $A_{i}$ is the Berry connection [7]

$$
A_{i}=i \hbar\left\langle\psi_{x} \mid \partial_{i} \psi_{x}\right\rangle
$$

$\partial_{i}$ denotes the partial derivative with respect to $x_{i}$, ' and the potential $V$ is the expectation value of the Hamiltonian in the state $\left|\psi_{x}\right\rangle$. The equation of motion derived from the Lagrangian is,

$$
f_{i j} \dot{x}_{j}=\partial_{i} V
$$

with

$$
f_{i j}=\partial_{i} A_{j}-\partial_{j} A_{i}
$$

Under the general condition that $f_{i j}$ is an everywhere invertible matrix (which in particular means that the space $\mathcal{M}$ is even-dimensional), a Poisson bracket can be defined and a symplectic structure introduced on $\mathcal{M}$. The bracket has the form [8]

$$
\{A, B\}=\left(f^{-1}\right)_{i j} \partial_{i} A \partial_{j} B
$$

and the equation of motion can then be written as

$$
\dot{x}_{i}=\left\{x_{i}, V\right\} .
$$

The corresponding symplectic form is,

$$
\omega=-\frac{1}{2} f_{i j} d x_{i} \wedge d x_{j}
$$

and in particular this determines the phase space volume. Thus, under the general conditions mentioned, a classical phase space can be derived from the quantum description. Note, however, that it is a generalized phase space in the sense that no configuration space has been identified.

The symplectic structure of the manifold $\mathcal{M}$ has a simple geometric interpretation. It is defined as the imaginary part of the scalar product in the

\footnotetext{
1 We use a shorthand notation by treating $x_{i}$ as a single parameter. In reality the phase space space for each particle will be multi-dimensional.
} 
tangent space of $\mathcal{M}$, which is obtained by projection from the Hilbert space of the full quantum system and can be written as

$$
f_{i j}=-2 \hbar \Im\left\{\left\langle D_{i} \psi_{x} \mid D_{j} \psi_{x}\right\rangle\right\}
$$

with $D_{i}$ the projected derivative,

$$
\left|D_{i} \psi_{x}\right\rangle=\left|\partial_{i} \psi_{x}\right\rangle-\left|\psi_{x}\right\rangle\left\langle\psi_{x} \mid \partial_{i} \psi_{x}\right\rangle
$$

Written in this form, it is manifest that the symplectic form, defining the classical kinetic energy, only depends on the properties of the projected subspace. The real part of the scalar product gives another, related structure on $\mathcal{M}$, which can be interpreted as a metric [9],

$$
g_{i j}=2 \hbar \Re\left\{\left\langle D_{i} \psi_{x} \mid D_{j} \psi_{x}\right\rangle\right\}
$$

This construction provides a natural way to introduce a metric on the phase space, and makes it possible to discuss its geometry.

At this point we will introduce an assumption about the geometrical structure of $\mathcal{M}$, which leads to a simplification in the discussion to follow. We assume $\mathcal{M}$ to be a Kähler manifold. This has several technical implications, but we will only use that there is a complex structure on $\mathcal{M}$, such that the symplectic and metric structures referred to above are the antisymmetric and symmetric part of the same complex Kähler metric. In terms of complex coordinates on $\mathcal{M}$, we then get the following expressions for the symplectic form and the metric,

$$
\begin{gathered}
\omega=-f_{\bar{z}_{i} z_{j}} d \bar{z}_{i} \wedge d z_{j}, \\
d s^{2}=-2 i f_{\bar{z}_{i} z_{j}} d \bar{z}_{i} d z_{j},
\end{gathered}
$$

with

$$
f_{\bar{z}_{i} z_{j}}=\partial_{\bar{z}_{i}} A_{j}-\partial_{z_{j}} A_{\bar{i}}
$$

This tensor can further be expressed as

$$
f_{\bar{z}_{i} z_{j}}=i \partial_{\bar{z}_{i}} \partial_{z_{j}} K(z, \bar{z})
$$

where $K(z, \bar{z})$ is the Kähler potential. 
The condition that $\mathcal{M}$ is a Kähler manifold is satisfied when the state vectors which define this manifold are, up to normalization, analytic functions of $z_{i}$,

$$
\left|\psi_{z}\right\rangle=\mathcal{N}(\bar{z}, z)\left|\phi_{z}\right\rangle,
$$

where $\left|\phi_{z}\right\rangle$ denotes the analytic part of the state vector and $\mathcal{N}(\bar{z}, z)$ is the normalization factor. The vector potentials are then given by

$$
\begin{aligned}
& A_{i}=-i \hbar \partial_{z_{i}} \ln \overline{\mathcal{N}}(\bar{z}, z), \\
& A_{\bar{i}}=i \hbar \partial_{\bar{z}_{i}} \ln \mathcal{N}(\bar{z}, z),
\end{aligned}
$$

and the Kähler potential is related in a simple way to the normalization factor,

$$
K(\bar{z}, z)=\hbar \ln |\mathcal{N}(\bar{z}, z)|^{-2} .
$$

\section{Coherent states of identical particles}

We now illustrate the general discussion by considering coherent states of the one dimensional harmonic oscillator, or equivalently, charged particles moving in two dimensions in the presence of a strong magnetic field that restricts the available states to the lowest Landau level. In this example we can explicitly derive the metric and symplectic structure, and show that they can be obtained from a Kähler potential.

We first define the coherent states for bosons and fermions and derive the corresponding classical mechanics. There is no unambiguous way to define coherent states for anyons, but we will use a construction which is very natural in this context, and again study the corresponding classical mechanics.

Since we are interested in the statistical mechanics, we also want to calculate the N-particle phase space volumes in the different cases. For this, it is necessary to start with a finite volume, and then take the thermodynamic limit. There are two obvious ways to confine the system, either by a potential, or by restricting the motion to a compact surface. In this section we shall consider the latter and study particles moving on a sphere. The case of a harmonic confining potential is treated in the Appendix. 


\section{A. Bosons and fermions in the plane}

We shall use the notation of ref. [10] and define a coherent state by translations of a minimum uncertainty reference state $|0\rangle$. The translation operators, $D(z)$, form a unitary and irreducible representation of the HeisenbergWeyl group, and in the following we shall use the following explicit representation in terms of creation and annihilation operators,

$$
D(z)=e^{z a^{\dagger}-\bar{z} a}=e^{-\frac{1}{2} \bar{z} z} e^{z a^{\dagger}} e^{-\bar{z} a},
$$

where $\left[a, a^{\dagger}\right]=1$, and $z$ is a dimensionless complex coordinate. In addition to the obvious relations $D(z)^{\dagger}=D(-z)$ and $D(0)=1$, we shall need the following multiplication rule,

$$
D\left(z_{1}\right) D\left(z_{2}\right)=e^{-\frac{1}{2}\left(\bar{z}_{1} z_{2}-\bar{z}_{2} z_{1}\right)} D\left(z_{1}+z_{2}\right) .
$$

The coherent states are now defined by,

$$
|z\rangle=D(z)|0\rangle=e^{-\frac{1}{2} \bar{z} z} e^{z a^{\dagger}}|0\rangle
$$

with a reference state $|0\rangle$ which is annihilated by $a$. For convenience we shall use a notation where the normalized coherent states are labeled by $z$ only, although the normalization factor also depend on $\bar{z}$. This is to distinguish the coherent states from the position eigenstates $|z, \bar{z}\rangle$, and should lead to no confusion. From (3.2) we immediately get the overlap between two coherent states,

$$
\left\langle z_{1} \mid z_{2}\right\rangle=\left\langle 0\left|D^{\dagger}\left(z_{1}\right) D\left(z_{2}\right)\right| 0\right\rangle=\left\langle 0\left|D\left(-z_{1}\right) D\left(z_{2}\right)\right| 0\right\rangle=e^{-\frac{1}{2}\left(\bar{z}_{1} z_{1}+\bar{z}_{2} z_{2}\right)+\bar{z}_{1} z_{2}} .
$$

An unsymmetrized basis of N-particle coherent states is defined by

$$
\begin{aligned}
|\mathbf{z}\rangle=\left|z_{1}, z_{2}, \ldots z_{N}\right\rangle & =D_{1}\left(z_{1}\right) D_{2}\left(z_{2}\right) \ldots D_{N}\left(z_{N}\right)|0,0, \ldots 0\rangle \\
& =e^{\left(-\frac{1}{2} \sum_{i=1}^{N} \bar{z}_{i} z_{i}\right)} e^{\left(\sum_{i=1}^{N} z_{i} a_{i}^{\dagger}\right)}|\mathbf{0}\rangle .
\end{aligned}
$$

Normalized N-particle coherent Bose and Fermi states are symmetric and antisymmetric linear combinations respectively,

$$
|\mathbf{z}, \pm\rangle=\left|z_{1}, z_{2}, \ldots z_{N}\right\rangle_{ \pm}=\mathcal{N}\left(z_{i}, \bar{z}_{i}\right) \frac{1}{\sqrt{N} !} \sum_{P} \eta_{P}^{ \pm} e^{z_{i} a_{i}^{\dagger}}|\mathbf{0}\rangle
$$


where sum is over permutations, $P$, and the sum in the exponent over the index $\mathrm{i}$ is suppressed. The permutation factor, $\eta_{P}$, equals 1 for bosons and \pm 1 for fermions depending on whether the permutation is even or odd. Note that all dependence on $\bar{z}_{i}$ is in the normalization factor $\mathcal{N}$, which is given by,

$$
|\mathcal{N}(\mathbf{z}, \overline{\mathbf{z}})|^{-2}=\sum_{P, P^{\prime}} \eta_{P} \eta_{P^{\prime}} \frac{1}{N !}\left\langle\mathbf{0}\left|e^{\bar{z}_{j^{\prime}} a_{j}} e^{z_{i_{P}} a_{i}^{\dagger}}\right| \mathbf{0}\right\rangle=\sum_{P} \eta_{P} e^{\bar{z}_{i_{P}} z_{i}}
$$

Following the general discussion in the previous section, we write the classical phase space Lagrangian (2.1) for the N-body system as,

$$
L(\mathbf{z}, \overline{\mathbf{z}})=\left\langle\mathbf{z}, \pm\left|i \hbar \partial_{t}-\hat{H}\right| \mathbf{z}, \pm\right\rangle
$$

where $\hat{H}$ is the quantum Hamiltonian. In the following we shall use the harmonic oscillator as a simple illustration, i.e. we take,

$$
\hat{H}=\hbar \omega \sum_{i=1}^{N}\left(a_{i}^{\dagger} a_{i}+\frac{1}{2}\right) .
$$

Using (3.8), (3.9) and (3.6) we get,

$$
L(\mathbf{z}, \overline{\mathbf{z}})=i \hbar\left(\dot{\bar{z}}_{i} \partial_{\bar{z}_{i}} \ln \mathcal{N}-\dot{z}_{i} \partial_{z_{i}} \ln \overline{\mathcal{N}}\right)-\hbar \omega z_{i} \partial_{z_{i}} \ln |\mathcal{N}|^{-2}-\frac{1}{2} N \hbar \omega .
$$

By varying with respect to $\bar{z}_{i}$ one easily verifies that the equation of motion is that of a harmonic oscillator, i.e. $\dot{z}_{i}=-i \omega z_{i}$. We can rewrite (3.10) on the standard form (2.2),

$$
L\left(z_{i}, \bar{z}_{i}\right)=\frac{1}{2}\left(A_{\bar{z}_{i}} \dot{\bar{z}}_{i}+A_{z_{i}} \dot{z}_{i}\right)-V\left(z_{i}, \bar{z}_{i}\right),
$$

and using the phase convention $\mathcal{N}=\overline{\mathcal{N}}$ the potentials $A_{z}$ and $A_{\bar{z}}$ and $V$ can all be obtained from the Kähler potential (2.17),

$$
\begin{aligned}
V(\mathbf{z}, \overline{\mathbf{z}}) & =\omega z_{i} \partial_{z_{i}} K(\mathbf{z}, \overline{\mathbf{z}}), \\
A_{i}(\mathbf{z}, \overline{\mathbf{z}}) & =\frac{i}{2} \partial_{z_{i}} K(\mathbf{z}, \overline{\mathbf{z}}), \\
A_{\bar{i}}(\mathbf{z}, \overline{\mathbf{z}}) & =-\frac{i}{2} \partial_{\bar{z}_{i}} K(\mathbf{z}, \overline{\mathbf{z}}),
\end{aligned}
$$


with

$$
K(\mathbf{z}, \overline{\mathbf{z}})=\hbar \ln |\mathcal{N}(\mathbf{z}, \overline{\mathbf{z}})|^{-2}=\hbar \sum_{P} \eta_{P} e^{\bar{z}_{i_{P}} z_{i}} .
$$

To get some insight into the meaning of these expressions we first analyze the two body case. Expressed in center of mass and relative coordinates, $Z=\frac{1}{2}\left(z_{1}+z_{2}\right)$ and $z=z_{1}-z_{2}$, the Kähler potential reads,

$$
K(Z, z, \bar{Z}, \bar{z})_{ \pm}=2 \bar{Z} Z+\ln \left[e^{\frac{1}{2} \bar{z} z} \pm e^{-\frac{1}{2} \bar{z} z}\right]
$$

where \pm refers to bosons and fermions respectively. The corresponding Lagrangians for the relative coordinate are now obtained using (3.11) - (3.14)

$$
\begin{aligned}
L_{B}(\bar{z}, z) & =\frac{i \hbar}{4}(\bar{z} \dot{z}-\dot{\bar{z}} z) \tanh \frac{\bar{z} z}{2}-\frac{\hbar \omega}{2} \bar{z} z \tanh \frac{\bar{z} z}{2} \\
L_{F}(\bar{z}, z) & =\frac{i \hbar}{4}(\bar{z} \dot{z}-\dot{\bar{z}} z) \operatorname{coth} \frac{\bar{z} z}{2}-\frac{\hbar \omega}{2} \bar{z} z \operatorname{coth} \frac{\bar{z} z}{2} .
\end{aligned}
$$

Note that fermionic Lagrangian is singular in the limit of small $r=\sqrt{\bar{z} z}$, i.e. when the particles come close together. This is however of no physical significance, since the singular piece is a total time derivative,

$$
\lim _{r \rightarrow 0} L_{F}=\frac{i \hbar}{2}(\bar{z} \dot{z}-\dot{\bar{z}} z) \frac{1}{\bar{z} z}=\frac{i \hbar}{2} \partial_{t} \ln (z / \bar{z}),
$$

which can be absorbed in $\mathcal{N}$ as a pure phase (relaxing the reality condition), or equivalently, as a pure gauge term in $A_{z}$ and $A_{\bar{z}}$. From (2.14) we get for the symplectic two form,

$$
\begin{aligned}
f_{\bar{z} z}^{B} & =\frac{i \hbar}{2}\left(\tanh \frac{\bar{z} z}{2}+\frac{\bar{z} z}{2 \cosh ^{2} \frac{\bar{z} z}{2}}\right) \stackrel{r \rightarrow \infty}{\rightarrow} \frac{i \hbar}{2}, \\
f_{\bar{z} z}^{F} & =\frac{i \hbar}{2}\left(\operatorname{coth} \frac{\bar{z} z}{2}-\frac{\bar{z} z}{2 \sinh ^{2} \frac{\bar{z} z}{2}}\right) \stackrel{r \rightarrow \infty}{\rightarrow} \frac{i \hbar}{2},
\end{aligned}
$$

where $z=r e^{i \phi}$. Note that although the Kähler potential is singular in the fermion case, the metric is well defined.

In both cases, in the limit of large $r$, we retain the naive flat metric, $d s^{2}=\hbar d z d \bar{z}$. Since we refer to relative coordinates this expression is reduced by a factor 2, as compared to the case of a single particle ${ }^{2}$. (Note that

\footnotetext{
${ }^{2}$ Our normalization is such, that for a harmonic oscillator hamiltonian with $m=$ $\omega=1, z$ is related to the usual coordinates and momenta by, $z=(x+i p) / \sqrt{2 \hbar}$, so $\hbar d z d \bar{z}=\left[(d x)^{2}+(d p)^{2}\right] / 2$.
} 
the appearance of $\hbar$ in these classical expressions is due to the use of the dimensionless coordinate $z$. As a more natural variable in the classical description we may use the dimensional coordinate $\tilde{z}=\sqrt{\hbar} z$ which then would remove $\hbar$ from the expressions.)

The more interesting limit is that of small $r$,

$$
\begin{aligned}
& d s^{2} \stackrel{r \rightarrow 0}{\rightarrow} \hbar\left[\rho^{2} d \theta^{2}+d \rho^{2}\right] \quad \text { (bosons) } \\
& d s^{2} \stackrel{r \rightarrow 0}{\rightarrow} \frac{\hbar}{3}\left[\rho^{2} d \theta^{2}+d \rho^{2}\right] \quad \text { (fermions), }
\end{aligned}
$$

where we have changed variables to $\rho=r^{2} / 2$ and $\theta=2 \phi$. We note that in these variables the metric has the standard flat-metric form in polar coordinates. Thus, the new angular variable is restricted to an interval of $2 \pi$ (for $\rho=0$ to be a regular point), and therefore $\phi$ is restricted to an interval of $\pi$. This has implications also for large separation of the particles, where the space of relative coordinates has the geometry of a cone rather than that of a plane. This is similar to the situation for the configuration space of two identical particles when this is constructed by identification of physically equivalent points [2]. However, in the present case the space is geometrically smooth for small separation and the reduction in volume (essentially by a factor 2) compared to that of non-identical particles appears naturally from the metric of the space and not through the identification (by hand) of equivalent points.

The phase space of bosons and fermions has the same flat metric for large separation. However, for small separation it is smooth but different in the two cases. This leads to a correction to the phase space volume coming from the short-distance behaviour which is different for bosons and fermions. If we fix the maximal separation, $R$ 3, of the particles, the volume of the interior of the selected region is determined from the form of the potential $A_{z}$ and $A_{\bar{z}}$ on the boundary alone. With the description still restricted to relative coordinates, the phase space volume becomesf

$$
V=-\int f_{\bar{z} z} d \bar{z} \wedge d z=-\left[\oint A_{z} d z+\oint A_{\bar{z}} d \bar{z}\right]
$$

\footnotetext{
${ }^{3} \mathrm{R}$ is again measured in dimensionsless units, just as $\mathrm{z}$

4 The phase space volume given by (3.19) is identical to the Berry phase associated with the interchange of the two particles. The close relation between phase space volume and Berry phase is the analogue of the two well-known aspects of quantum statistics: the symmetry of the wave function on one hand and the Pauli exclusion principle on the other.
} 
This gives in the two cases

$$
V_{B}=\frac{1}{2} \hbar \pi R^{2}, \quad V_{F}=\frac{1}{2} \hbar\left(\pi R^{2}-2 \pi\right)
$$

The difference in phase space volume is a manifestation of the the difference in statistics in the classical description. This is readily understood from a semiclassical description where the number of states in a (single particle) phase space is identical to the volume divided by $h$. (The factor $1 / 2$ in (3.20) follows from the fact that we refer to relative coordinates, with the angular integration in (3.19) running from 0 to $\pi$. The volume of one particle-space with the position of the second particle fixed would not include this factor.) In the following we will simply take the reduction in phase space volume due to the presence of the other particle as defining the statistics parameter in the classical description. We will then examine how the phase space volume of an $N$-particle system depends on this parameter, not only for bosons and fermions but also for intermediate values of the statistics parameter.

\section{B. Anyons in the plane}

It is well known that the harmonic oscillator coherent states states are identical to the maximally localized states of charged particles in a magnetic field projected to the lowest Landau level (LLL). The translation from harmonic oscillator to particle in the LLL is as follows

$$
\begin{aligned}
a & =\frac{i}{\sqrt{2}}\left(\tilde{\Pi}_{x}-i \tilde{\Pi}_{y}\right) \ell \\
z & =\frac{1}{\sqrt{2}}\left(R_{x}-i R_{y}\right) \frac{1}{\ell},
\end{aligned}
$$

where (in the symmetric gauge) $\tilde{\Pi}_{i}=p_{i}-\frac{1}{2} e B \epsilon_{i j} \hat{x}_{j}$ are the generators of magnetic translations, $R_{i}$ the guiding center coordinates, and $\ell=(\hbar / e B)^{\frac{1}{2}}$ the magnetic length. This relation just expresses that the configuration space of charged particles in the LLL is mathematically equivalent to the phase space of a particle in one dimension. The re-interpretation of the coherent states as describing particles in the lowest Landau level is helpful in two respects. We can in a simple way generalize the coherent state representation of bosons and fermions to fractional statistics, i.e. to anyons in the lowest Landau level. We can also more easily introduce a finite volume and take the correct 
thermodynamic limit. Although there is no natural way to restrict particle motion in one dimension to a finite phase space, charged particles moving in a finite area penetrated by a constant magnetic field, makes perfect sense. In particular we can (in theory) study anyons moving on a compactified space, like a sphere. This problem will be studied below, but first we generalize our coherent state formalism to the case of fractional statistics.

In complex coordinates, an N-body anyon wave function has the form,

$$
\Psi^{\nu}(\mathbf{z}, \overline{\mathbf{z}})=\prod_{i<j}\left(\frac{\bar{z}_{i}-\bar{z}_{j}}{z_{i}-z_{j}}\right)^{\nu / 2} \Psi_{B}(\mathbf{z}, \overline{\mathbf{z}}),
$$

where $\Psi_{B}$ is a totally symmetric function. In general very little is known about anyonic energy eigenstates for $N>2$. Exceptions are the LLL anyon states in a magnetic field which are of the form [11,

$$
\Psi_{\mathbf{m}}^{\nu}(\mathbf{z}, \overline{\mathbf{z}})=\prod_{i<j}\left(\bar{z}_{i}-\bar{z}_{j}\right)^{\nu} e^{-\frac{1}{2} \mathbf{z} \overline{\mathbf{z}}} S_{\mathbf{m}}(\overline{\mathbf{z}})
$$

where $\mathbf{m}=\left(m_{1}, \ldots m_{N}\right), m_{i}$ integer, and

$$
S_{\mathbf{m}}(\mathbf{z})=\mathcal{N}_{\mathbf{m}} \mathcal{S} \prod_{\mathbf{m}} z_{i}^{m_{i}}
$$

$\mathcal{S}$ is the symmetrization operator and $\mathcal{N}_{\mathbf{m}}$ a normalization constant. We now recall [12] that the fermion and boson coherent states in (3.6), up to a normalization factor, is nothing but the projection on the lowest Landau level of the appropriately (anti)symmetrized position eigenstates,

$$
|\mathbf{z}, \pm\rangle=C_{ \pm}(\mathbf{z}, \overline{\mathbf{z}}) P_{L L L}|\mathbf{z}, \overline{\mathbf{z}}, \pm\rangle,
$$

which implies that any bosonic of fermionic N-body wave function in the lowest Landau level can be expressed as

$$
\Psi^{ \pm}(\mathbf{z}, \overline{\mathbf{z}})=\left\langle\mathbf{z}, \overline{\mathbf{z}}, \pm\left|P_{L L L}\right| \Psi\right\rangle=C_{ \pm}(\mathbf{z}, \overline{\mathbf{z}})^{-1}\langle\mathbf{z}, \pm \mid \Psi\rangle
$$

In particular, if we are given a complete set $\Psi_{\mathbf{m}}^{ \pm}(\mathbf{z}, \overline{\mathbf{z}})$ of such LLL wave functions, we can reconstruct the N-particle coherent states by,

$$
\begin{aligned}
|\mathbf{z}, \pm\rangle & =C_{ \pm}(\mathbf{z}, \overline{\mathbf{z}}) \sum_{\mathbf{m}}|\mathbf{m}\rangle\langle\mathbf{m} \mid \mathbf{z}, \overline{\mathbf{z}}, \pm\rangle \\
& =C_{ \pm}(\mathbf{z}, \overline{\mathbf{z}}) \sum_{\mathbf{m}} \bar{\Psi}_{\mathbf{m}}^{ \pm}(\mathbf{z}, \overline{\mathbf{z}})|\mathbf{m}\rangle
\end{aligned}
$$

\footnotetext{
5 This can easily be verified by explicit calculation, and it is also natural since the coherent states are the minimum uncertainty states centered around $\mathbf{z}$.
} 
and using that the states $|\mathbf{m}\rangle$ are normalized, we can express the normalization $C_{ \pm}(\mathbf{z}, \overline{\mathbf{z}})$ as

$$
\left|C_{ \pm}(\mathbf{z}, \overline{\mathbf{z}})\right|^{-2}=\sum_{\mathbf{m}}\left|\Psi_{\mathbf{m}}^{ \pm}(\mathbf{z}, \overline{\mathbf{z}})\right|^{2}=\left\langle\mathbf{z}, \overline{\mathbf{z}} \pm\left|P_{L L L}\right| \mathbf{z}, \overline{\mathbf{z}}, \pm\right\rangle
$$

The same procedure can now be applied to anyons in the LLL provided that we define the coherent states by the projection of the position eigenstates on the LLL. This definition is not unique, but can be shown to have several good properties [12, 13]. By substituting (3.23) in the relations corresponding to (3.27) and (3.28) and redefining the normalization constant by an exponential and a Jastrow factor which is common for all the wave functions, we get

$$
|\mathbf{z}, \nu\rangle=\mathcal{N}_{\nu}(\mathbf{z}, \overline{\mathbf{z}}) \sum_{\mathbf{m}}|\mathbf{m}\rangle S_{\mathbf{m}}(\mathbf{z})
$$

with

$$
\left|\mathcal{N}_{\nu}(\mathbf{z}, \overline{\mathbf{z}})\right|^{-2}=\sum_{\mathbf{m}}\left|S_{\mathbf{m}}\right|^{2}=e^{\overline{\mathbf{z}} \mathbf{z}} \prod_{i<j}\left|z_{i}-z_{j}\right|^{-2 \nu}\left\langle\mathbf{z}, \overline{\mathbf{z}}, \nu\left|P_{L L L}\right| \mathbf{z}, \overline{\mathbf{z}}, \nu\right\rangle .
$$

These expressions are the anyonic counterparts to (3.6) and (3.7) in the case of bosons and fermions, and the derivation of the classical mechanics follows mutatis mutandis. The expressions are of course much more complicated than in the boson or fermion case, and there is no known analytic expression for the Kähler potential except in the case of two particles, where the polynomial part of the wavefunction in the relative coordinate $z$ is given by [11]

$$
S_{m}(z)=\frac{z^{2 m+\nu}}{\sqrt{\pi 2^{2 m} \Gamma(2 m+1+\nu)}} .
$$

The Kähler potential can then be calculated from (3.30) and expressed in terms of a generalized hypergeometric function,

$$
K(\bar{z}, z)=\hbar \ln \left[\frac{1}{\pi \Gamma(1+\nu)}{ }_{1} F_{2}\left(1 ; \frac{1}{2}+\frac{\nu}{2}, 1+\frac{\nu}{2} ; \frac{(\bar{z} z)^{2}}{16}\right)\right] .
$$

The large $r$ limit can be obtained from the properties of the hypergeometric function, and coincides with the result for bosons and fermions. The small $r$ limit can be read off directly from the leading term in (3.30),

$$
\lim _{r \rightarrow 0} K(\bar{z}, z)=\hbar \nu \ln \bar{z} z+\frac{\hbar}{2(1+\nu)(2+\nu)}\left(\frac{\bar{z} z}{2}\right)^{2}+\text { const }
$$


giving the asymptotic metric

$$
d s^{2} \stackrel{r \rightarrow 0}{\rightarrow}=\frac{2 \hbar}{(1+\nu)(2+\nu)}\left[\rho^{2} d \theta^{2}+d \rho^{2}\right]
$$

which interpolates smoothly between the expressions (3.18) for bosons and fermions. (Note that the phase space metric found in this way does not have the same physical dimension as the metric of the space in which the anyons move. Thus, there is a scale factor $\ell^{2} / \hbar$ between the two metrics, where $\ell$ is the magnetic length.)

In spite of the complicated form of the Kähler potential $K(\mathbf{z}, \overline{\mathbf{z}})$, there

is a simple property that immediately follows if we write it in the following form using the second identity in (3.30),

$$
K(\mathbf{z}, \overline{\mathbf{z}})=\hbar\left[\overline{\mathbf{z}} \mathbf{z}-2 \nu \sum_{i<j} \ln \left|z_{i}-z_{j}\right|+\ln \left\langle\mathbf{z}, \overline{\mathbf{z}}, \nu\left|P_{L L L}\right| \mathbf{z}, \overline{\mathbf{z}}, \nu\right\rangle\right] .
$$

For a translationally invariant state (corresponding to a constant magnetic field and no external potential) only the first term can depend on the CM coordinate $Z$. Equivalently, we consider the limit $z_{i}=Z$ where the positions of all the particles coincide, and get

$$
K(Z, \bar{Z})=N \hbar \bar{Z} Z
$$

corresponding to a single particle of charge $N$, as expected. Below we shall see that this relation is altered when the particles are moving on a sphere, and the corresponding expression will allow us to calculate the pertinent phase space volume for particles with different statistics.

\section{B. Identical particles on a sphere}

In this section we will calculate the $N$-particle phase space volume for particles confined to a finite region. We are interested in the dependence of the volume on the particle statistics. This extends the previous discussion of the two-particle case and makes it possible to derive the (classical) statistical mechanics of the particles. As a convenient regularization of the system size, we consider a phase space with spherical geometry!

${ }^{6}$ The most natural choice for a closed two-surface seems to be the torus, since the sphere has a finite curvature. There is, however, a technical difficulty in generalizing the results in the plane to a torus in that the magnetic translation operators are not well defined for general translations due to the periodicity conditions [15]. 
A charged particle moving on a unit sphere, penetrated by $2 j$ units of magnetic flux has a total angular momentum $J=j+L$ where $L$ is the orbital angular momentum. The lowest Landau level corresponds to $L=0$ and has a $2 j+1$ degeneracy [14]. Again we can construct coherent states by acting on an arbitrary minimal uncertainty state, that we shall take to be $|j,-j\rangle$, with the appropriate group elements of $\mathrm{SU}(2)$. We describe the sphere by stereographic projection and use a dimensonless complex coordinate, $z$, related to the polar angles by, $z=-\tan (\theta / 2) e^{-i \phi}$. It is easy to show that this $z$ is translated into the dimensionless $z$ introduced earlier in (3.21) by the substitution $z \rightarrow \sqrt{\frac{1}{2 j}} z$. For ease of notation, we shall make this substitution only in the final expressions.

The $\mathrm{SU}(2)$ generators in the $j$-representation, and the corresponding coherent states are given by,

$$
D(z)=e^{z J_{+}} e^{\eta J_{0}} e^{-\bar{z} J_{-}}
$$

where $J_{i}$ satisfy the standard angular momentum commutation relations and $\eta=\ln (1+\bar{z} z)$. The $D(z)$ :s satisfy a multiplication rule similar to (3.2), but here it is sufficient to know the overlap,

$$
\langle z \mid w\rangle=[(1+\bar{z} z)(1+\bar{w} w)]^{-j}(1+\bar{z} w)^{2 j} .
$$

Note that in the limit $j=R^{2} / l^{2} \rightarrow \infty$ corresponding to a large radius, or a strong magnetic field, we recover,

$$
\langle z \mid w\rangle \rightarrow e^{-\frac{1}{2} \bar{z} z-\frac{1}{2} \bar{w} w+\bar{z} w}
$$

where we have rescaled $z \rightarrow \sqrt{\frac{1}{2 j}} z$. We can now immediately take over the results (3.6) and (3.7) for the bosonic and fermionic states in the plane,

$$
|\mathbf{z}, \pm\rangle=\mathcal{N}(\mathbf{z}, \overline{\mathbf{z}}) \frac{1}{\sqrt{N !}} \sum_{P} \eta_{P}^{ \pm} e^{z_{i_{P}} J_{+}^{i}}|\mathbf{0}\rangle
$$

and their normalization

$$
|\mathcal{N}(\mathbf{z}, \overline{\mathbf{z}})|^{-2}=\sum_{P, P^{\prime}} \eta_{P} \eta_{P^{\prime}} \frac{1}{N !}\left\langle\mathbf{0}\left|e^{\bar{z}_{j_{P^{\prime}}} J_{-}^{j}} e^{z_{i_{P}} J_{+}^{i}}\right| \mathbf{0}\right\rangle .
$$


Using (3.37) and (3.38) we can easily calculate the relevant overlap,

$$
\left\langle\mathbf{0}\left|e^{\bar{z} J_{-}^{j}} e^{w J_{+}^{i}}\right| \mathbf{0}\right\rangle=\delta_{i j}(1+\bar{z} w)^{2 j},
$$

SO

$$
|\mathcal{N}(\mathbf{z}, \overline{\mathbf{z}})|^{-2}=\sum_{P} \eta_{P} \prod_{i}\left(1+\bar{z}_{i_{P}} z_{i}\right)^{2 j}
$$

For the case of $\mathrm{N}$ coinciding bosons, $z_{i}=z$, we immediately get the following Kähler potential

$$
K(z, \bar{z})=N \hbar 2 j \ln \left(1+\frac{\bar{z} z}{2 j}\right)
$$

and the corresponding metric

$$
d s^{2}=\frac{2 N \hbar}{\left(1+\frac{\bar{z} z}{2 j}\right)^{2}} d z d \bar{z}
$$

is just $N$ times that of a sphere. Following Manton [6], we can use this result to obtain the the volume of the N-boson phase space 7 . The essential observation is that the submanifold spanned by the configurations of $\mathrm{N}$ coinciding bosons is a complex curve of degree $\mathrm{N}$ in the manifold $C P_{N}$. The metric (3.45) immediately gives the volume corresponding to this complex curve, which can be shown to be $\mathrm{N}$ times the area, $A$, obtained by integrating the fundamental two form, $\omega$, in (2.12) over a complex line. It then follows from a general theorem for Kähler manifolds that the total volume is given by,

$$
V=\frac{1}{N !}(A)^{N} .
$$

In our case, using (3.44) and (2.12), we get

$$
A=\hbar \int_{s p h} \omega=h 2 j=\frac{\hbar 4 \pi R^{2}}{l^{2}}=e \Phi,
$$

\footnotetext{
${ }^{7}$ In [16] a formula for the volume of the moduli space for vortices moving on an arbitrary genus Riemannian surface is derived making extensive use of theorems from complex geometry
} 
which shows that the area is $h$ times the number of flux quanta $\phi_{0}=h / e$ which penetrate the sphere, or equivalently, $\hbar$ times the area in units of $l^{2}$.

In the fermion case, we first note from (3.43) that the normalization can be expressed as a determinant,

$$
|\mathcal{N}(\mathbf{z}, \overline{\mathbf{z}})|^{-2}=N ! \operatorname{det}\left(1+\bar{z}_{i} z_{j}\right)^{2 j} .
$$

In this case we cannot directly put the particles on top of each other (that would give a diverging $\mathcal{N}$ ), so we instead put $z_{i}=z+\delta_{i}$ and consider the limit $\delta_{i} \rightarrow 0$. Expanding in $\delta_{i}$, we get,

$$
\operatorname{det}\left(1+\bar{z}_{i} z_{j}\right)^{2 j} \simeq(1+\bar{z} z)^{2 j N} \operatorname{det} M_{i j}
$$

where

$$
M_{i j}=1+\frac{2 j}{(1+\bar{z} z)}\left(\bar{z} \delta_{i}+z \bar{\delta}_{j}\right)
$$

The matrix $M$ is hermitian, so the determinant is real and furthermore it has zeros for $\delta_{i}=\delta_{j}$. These properties together with power counting is sufficient to establish

$$
\operatorname{det} M_{i j}=C\left(\frac{\bar{z} z}{(1+\bar{z} z)^{2}}\right)^{\frac{N(N-1)}{2}} \prod_{i<j}\left|\delta_{i}-\delta_{j}\right|^{2},
$$

where $C$ is a $z$ and $\delta$ independent constant. Combining (3.49) and (3.51) we get, up to a constant, the Kähler potential

$$
\begin{aligned}
K(z, \bar{z}) & =\ln (1+\bar{z} z)^{2 j N-N(N-1)}+\ln (\bar{z} z)^{\frac{N(N-1)}{2}} \\
& \rightarrow 2 j N\left(1-\frac{N-1}{2 j}\right) \ln \left(1+\frac{\bar{z} z}{2 j}\right)-\frac{1}{2} N(N-1) \ln (\bar{z} z)
\end{aligned}
$$

The last term can be removed by a so called Kähler gauge transformation, and corresponds to redefining the normalization constant by an analytic factor that does not contribute to the metric. The first term differs from the boson case (3.44) only by the "reduction" factor $1-(N-1) / 2 j$ which equals one for $N=1$, corresponding to a single fermion, and zero for $N=2 j+1$ corresponding to a filled Landau level. Using the same argument as in the boson case, we get the phase space volume,

$$
V_{F}=\frac{1}{N !}(A-(N-1) h)^{N} .
$$


Again the interpretation is clear - the available phase space for one particular fermion is reduced with an amount $h$ by each of the other particles present in the system. This is consistent with the semi-classical interpretation, where each quantum state is associated with a phase space volume $h$. Note that there is a maximum number of particles allowed, $N=2 j+1$, in which case the phase space volume (3.53) vanishes. This corresponds to the situation where all the lowest angular momentum states are filled, i.e. to a filled lowest Landau level. Thermodynamically this state is interpreted as being incompressible, as we will discuss further in Sect. 5 .

Finally we consider the case of anyons. A complete set of LLL anyon wave functions on the sphere corresponding to (3.23) in the plane is given by,

$$
\Psi_{\mathbf{m}}^{\nu}(\mathbf{z}, \overline{\mathbf{z}})=\prod_{i<j}\left(\bar{z}_{i}-\bar{z}_{j}\right)^{\nu} \prod_{i}\left(1+z_{i} \bar{z}_{i}\right)^{-j+\frac{\nu}{2}(N-1)} S_{\mathbf{m}}(\overline{\mathbf{z}}) .
$$

Following the steps leading from (3.23) to (3.30), we get the anyonic version for (3.35),

$$
\begin{aligned}
K(\mathbf{z}, \overline{\mathbf{z}}) & =\hbar\left(-j+\frac{\nu}{2}(N-1)\right) \sum_{i} \ln \left(1+\frac{z_{i} \bar{z}_{i}}{2 j}\right) \\
& -2 \hbar \nu \sum_{i<j} \ln \left|z_{i}-z_{j}\right|+\hbar \ln \left\langle\mathbf{z}, \overline{\mathbf{z}}, \nu\left|P_{l l l}\right| \mathbf{z}, \overline{\mathbf{z}}, \nu\right\rangle .
\end{aligned}
$$

Note that in this case we do not have any explicit expression for the Kähler potential corresponding to (3.41) in the case of bosons and fermions. We can, however, again deduce the metric corresponding to configurations with coinciding anyons, i.e. $z_{i}=z$, from the above expression, again noting that the complicated overlap in the last term must be independent of $z$, this time because of rotational invariance. The Kähler potential becomes,

$$
K(z, \bar{z})=\hbar N 2 j\left(1-\frac{\nu(N-1)}{2 j}\right) \ln \left(1+\frac{2 \bar{z} z}{j}\right)+\ldots
$$

which again smoothly interpolates between the bosonic $(\nu=0)$ and fermionic $(\nu=1)$ results,

$$
V_{\nu}=\frac{1}{N !}(A-\nu(N-1) h)^{N} .
$$


The expressions we have found above for the $N$-particle phase space volume demonstrates a "classical exclusion principle". Thus, each new particle introduced in the system will find the available volume reduced by $\alpha=\nu h$ relative to the previous one. The quantity $\alpha$, i.e. the reduction in phase space volume, can be taken as defining the classical statistics parameter of the particles. In the present case it is simply the (dimensionless) quantum statistics parameter $\nu$ multiplied with Planck's constant $h$. In other cases

such a classical statistics parameter may be possible to define in terms of reduced phase space volume, even if there is no underlying point particle description. In the next section we will study such an example.

\section{Vortex statistics}

In the previous sections we have discussed how a phase space description, with a classical statistics parameter, can be derived from a (constrained) quantum description. In this section we will consider a somewhat different system; a classical field theory with soliton solutions. The system we have in mind is the Chern-Simons Ginzburg-Landau (CSGL) theory, originally introduced as a field theory for the quantum Hall effect [17], with vortices (quasi-particles) as soliton solutions. In a certain approximation the dynamics can be described in terms of vortex coordinates alone, and a phase space description can be derived from the full theory. In this description the vortices will be associated with a non-trivial classical statistics parameter, and we will show that the value of this parameter agrees with the value of the (quantum) fractional statistics parameter usually associated with quasiparticles of the quantum Hall effect. In this derivation we will make use of the close connection which exists between the CSGL Lagrangian and the Lagrangian of the relativistic abelian Higgs model discussed by Samols, Manton and others 15, 6, 18]. The metric of the vortex space is the same in these two cases and it is Kähler [5]. We make use of the results of Manton [6] for the volume of the $N$-vortex space, to determine the classical statistics parameter of the vortices.

The field theory Lagrangian, which describes fields in a 2+1-dimensional space time, is

$$
L=\int d^{2} x\left[i \hbar \phi^{*} D_{0} \phi-\frac{\hbar^{2}}{2 m}|\vec{D} \phi|^{2}-\frac{\lambda}{4}\left(|\phi|^{2}-\rho_{0}\right)^{2}+\mu \hbar \epsilon^{\mu \nu \rho} a_{\mu} \partial_{\nu} a_{\rho}\right]
$$


where $\phi$ is a complex matter field, $a_{\mu}$ a Chern-Simons field, $m$ a mass parameter, $\lambda$ the interaction strength, $\rho_{0}$ the preferred density of the system and $\mu$ a statistics parameter. In this classical field theory $\hbar$ only plays the role of a dimensional parameter. For the original Laughlin quantum Hall states described by the model the statistics parameter takes the values $\mu=1 /[4 \pi(2 k+1)]$ with integer $k . D_{0}$ and $\vec{D}$ denote covariant derivatives

$$
\begin{aligned}
D_{0} & =\frac{\partial}{\partial t}+i a_{0} \\
\vec{D} & =\nabla-i \vec{A}
\end{aligned}
$$

with

$$
\vec{A}=\vec{a}+\frac{e}{\hbar} \vec{A}_{e x t}
$$

$\vec{A}_{\text {ext }}$ describes a constant external magnetic field, $B_{\text {ext }}$, which we assume is adjusted to fit the parameter $\rho_{0}$ so that the ground state is described by a constant field $\phi$ of density $\rho_{0}$ with vanishing effective magnetic field, $B=b+\frac{e}{\hbar} B_{e x t}=0$. The physical interpretation is that the system is at (or close to) the center point of a quantum Hall plateau.

It is convenient to change to dimensionless form,

$$
L=\int d^{2} x\left[i \phi^{*} D_{0} \phi-\frac{1}{2}|\vec{D} \phi|^{2}-\frac{\tilde{\lambda}}{4}\left(|\phi|^{2}-1\right)^{2}+\epsilon^{\mu \nu \rho} a_{\mu} \partial_{\nu} a_{\rho}\right]
$$

where the new Lagrangian is obtained from the original one by the substitutions,

$$
\begin{aligned}
\phi & \rightarrow \sqrt{\rho_{0}} \phi, \\
A_{0} & \rightarrow \frac{\hbar \rho_{0}}{\mu m} A_{0}, \quad \vec{A} \rightarrow \sqrt{\frac{\rho_{0}}{\mu}} \vec{A}, \\
\vec{r} & \rightarrow \sqrt{\frac{\mu}{\rho_{0}}} \vec{r}, \quad t \rightarrow \frac{\mu m}{\hbar \rho_{0}} t, \\
L & \rightarrow \hbar^{2} \frac{\rho_{0}}{m} L .
\end{aligned}
$$

8 Although the theory is non-relativistic, we choose to use a relativistic notation for the CS field with $a_{0}=a^{0}, a_{i}=-a^{i}(\mathrm{i}=1,2)$ and $b=\epsilon_{i j} \partial_{i} a^{j}$. 
The single dimensionless parameter in the rescaled Lagrangian is $\tilde{\lambda}=\frac{\mu m}{\hbar^{2}} \lambda$.

There is no independent dynamics associated with the Chern-Simons field, since variation with respect to $a_{0}$ gives a relation between magnetic field and (charge) density, which can be written as,

$$
B+\left(\frac{1}{2} \rho-B_{\text {ext }}\right)=0
$$

with $\rho=|\phi|^{2}$. We assume $B_{\text {ext }}=1 / 2$ to give $\rho=1$ and $B=0$ in the ground state (all fields in dimensionless form). For finite energy configurations these values are reached asymptotically. With this assumption the constraint equation (4.6) for $B$ is rewritten as,

$$
B+\frac{1}{2}(\rho-1)=0
$$

For stationary states the energy can be expressed as,

$$
E=\int d^{2} x\left[\frac{1}{2}\left|D_{+} \phi\right|^{2}+\frac{1}{2} B+(\tilde{\lambda}-1) B^{2}\right] .
$$

with $D_{+} \phi=\left(D_{1}+i D_{2}\right) \phi$. The energy has the lower bound

$$
E \geq \int d^{2} x \frac{1}{2} B=N \pi
$$

where $N$ is a non-negative integer. For the special value $\tilde{\lambda}=1$ of the coupling the lower bound can be saturated. The field $\phi$ then satisfies the linear differential equation

$$
D_{+} \phi=0
$$

The two equations (4.7) and (4.10) define (for $\tilde{\lambda}=1$ ) stationary vortex configurations with $N$ vortices of equal circulation [19] ?. Since the configurations (for fixed $N$ ) are degenerate in energy, the vortices can be regarded as non-interacting. Gauge-equivalent configurations may naturally be considered as physically equivalent, and the vortex space can then be identified with the (moduli) space obtained from the space of field configurations after

\footnotetext{
${ }^{9}$ We have chosen $B_{\text {ext }}$ to be positive. With opposite sign the lower bound is saturated with vortices of opposite circulation.
} 
the identification of gauge-equivalent configurations [5]. A point in vortex space is identified by the set of $N$ (unordered) vortex coordinates which corresponds to zeros of the field $\phi$.

For values of $\tilde{\lambda}$ close to 1 the low energy configurations correspond to slowly moving vortices. A meaningful approximation is then to impose (4.7) and (4.10) as constraints on the field configurations. The constrained fields describe a system of weakly interacting vortices. In the $a_{0}=0$ gauge the Lagrangian takes the form,

$$
L=\int d^{2} x\left[i \phi^{*} \dot{\phi}+\dot{\vec{A}} \times\left(\vec{A}-\vec{A}_{e x t}\right)-(\tilde{\lambda}-1) B^{2}\right]-N \pi .
$$

Due to (4.7) this Lagrangian is invariant (up to a total time derivative) under time dependent gauge transformations, $\vec{A} \rightarrow \vec{A}+\nabla \xi, \phi \rightarrow e^{i \xi} \phi$, and can therefore be interpreted as the vortex Lagrangian defined on the space of gauge-equivalent field configurations.

It is useful to consider the two fields $\phi$ and $\vec{A}$ as components of a complex two-component field [5],

$$
u=\left(\begin{array}{c}
\phi \\
A
\end{array}\right)
$$

with $A$ as the complex field $A=A_{1}+i A_{2}$. A hermitian scalar product is introduced as

$$
\langle u \mid v\rangle=\int d^{2} x u^{\dagger} v
$$

With this notation, up to total time derivatives the kinetic term in the Lagrangian (i.e. the part with time derivatives) can be written as

$$
T=i\left\langle u-u_{e x t} \mid \dot{u}\right\rangle
$$

with $u_{\text {ext }}=\left(0, A_{\text {ext }}\right)$.

The vortex configurations are described by a set of vortex coordinates $x=$ $\left\{x_{1}, x_{2}, \ldots, x_{n}\right\}$, with $x_{i}$ corresponding to two real or one complex coordinate of vortex $i$. The precise form of the multi-vortex configurations for given coordinates is not known, but their existence is [19]. The kinetic term for these configurations can be written

$$
T=\mathcal{A}_{i}(x) \dot{x}_{i}
$$


where the new vector potential is

$$
\begin{aligned}
\mathcal{A}_{i} & =i\left\langle u-u_{\text {ext }} \mid \partial_{i} u\right\rangle \\
& =i\left\langle u \mid \partial_{i} u\right\rangle-\partial_{i}\left\langle u_{\text {ext }} \mid u\right\rangle,
\end{aligned}
$$

and where the differentiation now is with respect to the vortex coordinates. The corresponding field tensor which defines the symplectic form and the phase space structure of the vortex space is

$$
\mathcal{F}_{i j}=\partial_{i} \mathcal{A}_{j}-\partial_{j} \mathcal{A}_{i}=-2 \Im\left\langle\partial_{i} u \mid \partial_{j} u\right\rangle
$$

The vortex space is the space of gauge equivalent field configurations which satisfy (4.7) and (4.10). Let us re-consider this gauge equivalence in terms of the complex fields $u$. The infinitesimal gauge transformations have the form

$$
\delta_{v} u=\left(i \chi \phi, 2 \partial_{\bar{z}} \chi\right)
$$

with $\chi$ a real function, so the vectors $\delta_{v} u$ define a real vector space. This can be extended to a complex vector space if $\chi$ is allowed to be complex. We will refer to the corresponding transformations as complex gauge transformations, with $\Gamma(u)$ as the projection onto this complex subspace, and we will refer to the directions defined by the gauge transformations (4.18) as vertical directions. The orthogonal directions (horizontal directions) are defined by variations in the field $u$,

$$
\delta_{h} u=(\delta \phi, \delta A),
$$

which satisfy $\left\langle\delta_{v} u \mid \delta_{h} u\right\rangle=0$, implying

$$
2 \partial_{z} \delta A+i \phi^{*} \delta \phi=0
$$

The real part is

$$
\nabla \cdot \delta \vec{A}+\frac{i}{2}\left(\phi^{*} \delta \phi-\phi \delta \phi^{*}\right)=0
$$

and the imaginary part is

$$
\delta B+\frac{1}{2} \delta \rho=0
$$


Changes in the fields which follows from variations in the vortex coordinates will automatically satisfy the second equation, (4.22), due to the constraint (4.7). The first equation, (4.21), can be satisfied provided we make use of the freedom to include real gauge transformations in the variations of the fields. Thus, we may assume both these equations, or equivalently (4.20) to be satisfied when $\delta A$ and $\delta \phi$ are replaced by the corresponding derivatives with respect to vortex positions.

We introduce $\Pi(u)=I-\Gamma(u)$ as the projection onto the horizontal directions and $D_{i}=\Pi \partial_{i}$ as the projected derivative. With the assumption that the gauge condition (4.21) is satisfied the vector potential $\mathcal{A}_{i}$ can be written as

$$
\mathcal{A}_{i}=i\left\langle u-u_{\text {ext }} \mid D_{i} u\right\rangle .
$$

It transforms under a (complex) gauge transformation $\chi$ as

$$
\mathcal{A}_{i} \rightarrow \mathcal{A}^{\prime}{ }_{i}=\mathcal{A}_{i}-\partial_{i} \Theta .
$$

with

$$
\Theta=\int d^{2} x\left[\phi^{*} \phi+2 i \partial_{\bar{z}}\left(A^{*}-A_{e x t}^{*}\right)\right] \chi .
$$

This means that $\mathcal{F}_{i j}$ is invariant under complex gauge transformations and can be written as

$$
\mathcal{F}_{i j}=-2 \Im\left\langle D_{i} u \mid D_{j} u\right\rangle,
$$

since the difference between $\partial_{i} u$ and $D_{i} u$ can (locally) be eliminated by a (vortex-position dependent) gauge transformation.

We may consider the quantity

$$
\eta_{i j}=\left\langle D_{i} u \mid D_{j} u\right\rangle
$$

as defining a hermitian metric tensor for the vortex space. It is obtained from the scalar product (4.13) by projection on the horizontal directions. The tensor $\mathcal{F}_{i j}$, which is derived from the kinetic part of the Lagrangian, and which defines the phase space structure of the vortex space, can now be identified with the imaginary part of this metric tensor. 
The metric (4.27) is relevant also for the relativistic abelian Higgs model, as we shall now demonstrate. The Lagrangian of this model has the form

$$
L=\int d^{2} x\left[\frac{1}{2}\left(D_{\mu} \phi\right)^{*} D^{\mu} \phi-\frac{1}{4} F_{\mu \nu} F^{\mu \nu}-\frac{2 \tilde{\lambda}-1}{8}\left(|\phi|^{2}-1\right)^{2}\right] .
$$

It is quadratic in time derivatives and has the Chern-Simons field replaced by a Maxwell field. The energy has the same lower bound (4.9) as the GLCS model, and for $\tilde{\lambda}=1$ this lower limit is saturated if both the equations (4.7) and (4.10), known as the Bogomolny equations, are satisfied. Thus, if these equations are used to define the $N$-vortex space, the vortex space is the same for the two models. However, the kinematics, as defined by the kinetic part of the Lagrangian is not the same in the two cases. The non-relativistic model is linear in time derivatives, which means that the vortex space has the character of a phase space, while the relativistic model is quadratic in time derivatives, which gives the vortex space the character of a configuration space.

Expressed in the $A_{0}=0$ gauge and constrained by the Bogomolny equations, the Lagrangian of the Higgs model has the form

$$
L^{\prime}=T^{\prime}-V
$$

with

$$
\begin{aligned}
T^{\prime} & =\frac{1}{2} \int d^{2} x\left[\dot{\phi}^{*} \dot{\phi}+\dot{\vec{A}} \cdot \dot{\vec{A}}\right], \\
V & =\int d^{2} x(\tilde{\lambda}-1) B^{2}+N \pi,
\end{aligned}
$$

and the fields constrained by Gauss' law

$$
\nabla \cdot \dot{\vec{A}}+\frac{i}{2}\left(\phi^{*} \dot{\phi}-\dot{\phi}^{*} \phi\right)=0
$$

The potential $V$ is the same, but the kinetic term $T^{\prime}$ is different from that of the CSGL model. We note that the constraint (4.31) corresponds to the real part (4.21) of the condition for motion in horizontal direction. Also the imaginary part (4.22) is satisfied due to the Bogomolny equations. The constraint on the motion, given by Gauss' law, can be expressed in terms of 
the projection $\Pi$ and the field $u$ as

$$
\begin{aligned}
T^{\prime} & =\frac{1}{2}\langle\dot{u}|\Pi| \dot{u}\rangle \\
& =\frac{1}{2} \dot{x}_{i} \dot{x}_{j}\left\langle D_{i} u \mid D_{j} u\right\rangle .
\end{aligned}
$$

The kinetic term $T^{\prime}$ is different, but related to the kinetic term $T$ of the CSGL model. $T^{\prime}$ is determined by the real part, whereas $T$ is determined (up to a gauge transformation) by the imaginary part of the same hermitian metric (4.27). This metric has been examined in some detail by Samols [5] for the case of the abelian Higgs model. It is a Kähler metric, which is conveniently expressed in terms of complex vortex coordinates as

$$
d s^{2}=-2 i \mathcal{F}_{\bar{z}_{i} z_{j}} d \bar{z}_{i} d z_{j}
$$

with

$$
\mathcal{F}_{\bar{z}_{i} z_{j}}=i\left[\left\langle D_{\bar{z}_{i}} u \mid D_{z_{j}} u\right\rangle-\left\langle D_{z_{j}} u \mid D_{\bar{z}_{i}} u\right\rangle\right]
$$

The corresponding Kähler 2-form is

$$
\omega=-\mathcal{F}_{\bar{z}_{i} z_{j}} d \bar{z}_{i} \wedge d z_{j}
$$

The Kähler form determines the symplectic structure and the volume of the vortex space. This $(2 N$-dimensional) volume is the same whether the vortex space is considered as a configuration space (i.e. with the volume determined by the real part of the metric) or as a phase space (with the volume determined by the imaginary part), and has been calculated by Manton [6] for $N$ vortices on a sphere. The result is $\square$

$$
V_{N}=\frac{1}{N !}\left(A-8 \pi^{2}(N-1)\right)^{N}
$$

with $A$ as the volume (area) of the one-vortex space. The volume (4.36) has the same form as discussed in Sect. 3 for $N$ identical particles with non-trivial

\footnotetext{
10 Manton's result has been changed with a factor $(2 \pi)^{N}$ to fit the definition of the metric in this paper. We also express the $N$-vortex volume in terms of the single-vortex volume $A$ rather than the area of the sphere. This gives a factor $N-1$ instead of $N$ in the second term of Eq.(4.36).
} 
classical statistics. With the vortex space interpreted as a phase space, the statistics of the vortices can be extracted from the reduction term $8 \pi^{2} N$. In order to do so correctly we have to re-write the volume (4.36) in dimensional form. The phase space volume is determined by the Lagrangian (4.4), and as follows from the transformations (4.5) the phase space dimensions are correctly re-introduced by the substitutions $A \rightarrow \mu \hbar A$ and $V_{N} \rightarrow(\mu \hbar)^{N} V_{N}$. In dimensional form the expression for the $N$-vortex volume is

$$
V_{N}=\frac{1}{N !}(A-4 \pi \mu h(N-1))^{N}
$$

and the classical statistics parameter as determined by the reduction in available phase space due to the presence of other vortices therefore is

$$
\alpha=4 \pi \mu h \equiv g h .
$$

We can interpret $g$, the classical parameter divided by $h$, as the dimensionless quantum statistics parameter. The value $g=4 \pi \mu$ agrees with the value of the statistics parameter as determined from Berry phase calculations with Laughlin wave functions [20], or from the properties of vortices in the CSGL model [17].

\section{Statistical mechanics}

\section{A. Entropy and pressure of identical particles}

We have already emphasized that even if the classical equations of motion, and thus the classical dynamics, does not depend on the classical statistics parameter $\alpha$, the statistical mechanics (and thus the thermodynamics), does. In this section we demonstrate this by first calculating the entropy and pressure in the two model systems considered in sections 3 and 4, charged particles in the lowest Landau level, and vortices in the CSGL model respectively. The results of this calculation fit nicely into the general framework of "fractional exclusion statistics" for particles with degenerate energy levels, and we briefly review the basics of this topic before further discussing our results.

We assume the interaction strength to be $\tilde{\lambda}=1$ for the CSGL theory. The vortex system is then degenerate in energy. That is also the case for a system of anyons in the lowest Landau level. Thus, both these systems 
have the special property that the energy does not depend on the state, but only on the number of particles. This means that the statistical mechanics is determined by the phase space volume $V_{N}$, which has been determined in the previous sections, and by the energy $E_{N}$. The classical partition function is simply the total number of states, $V_{N} / h^{N}$ multiplied with the Boltzmann factor, i.e.

$$
Z_{N}=\frac{V_{N}}{h^{N}} e^{-\beta E_{N}}
$$

The following simple expressions for the free energy $F$ and the entropy $S$, immediately follow,

$$
\begin{aligned}
F & =E_{N}-T \ln \left(V_{N} / h^{N}\right), \\
S & =\ln \left(V_{N} / h^{N}\right) .
\end{aligned}
$$

where is Boltzmann's constant is set to unity. The pressure is usually defined by $P=-(\partial F / \partial \mathcal{V})_{T}$, where $\mathcal{V}$ is the volume of real space, but in the systems we have considered the real two-dimensional space where the particles or vortices move is proportional to the phase space, so we simply define the pressure as,

$$
P=-\left(\frac{\partial F}{\partial A}\right)_{T}=T \frac{\partial \ln \left(V_{N} / h^{N}\right)}{\partial A}
$$

where $A=V_{1}$ is the phase space volume for a single particle. Substituting the results (3.57) or (4.37), we get

$$
\begin{aligned}
S & =N \ln (1-\alpha \rho)+N \ln \frac{A}{h}-N \ln N+N, \\
\beta P & =\frac{\rho}{1-\alpha \rho},
\end{aligned}
$$

where $\alpha=\nu h$ or $g h$, and where we have introduced the classical phase space density $\rho=N / A$ and neglected the difference between $N$ and $N-1$, which is irrelevant in the thermodynamic limit.

The expression (5.5) shows that there is a maximum density $\rho=1 / \alpha$ allowed by the system, which corresponds to an infinite pressure and therefore to an incompressible state. For the phase space volume this means $V_{N}=0$, 
i.e. there is no available phase space volume for any new particle added to the system. For the anyon system this situation corresponds to a completely filled Landau level. What is unusual about this is that the blocking, which can be interpreted as representing a generalized Pauli principle, shows up not only in the quantum but also in the classical description of the system.

\section{B. Exclusion statistics and the classical limit}

The generalization of the Pauli exclusion principle introduced by Haldane [四], usually called exclusion statistics, states that in the presence of particles in a set of given quantum states, the number of available one-particle states for any new particle added to the system is reduced. More precisely, the addition of $\Delta N$ particles changes the number of available states, $d_{N}$ according to

$$
\Delta d_{N}=-g \Delta N
$$

where $g$ is the exclusion statistics parameter. The statistical weight, or number of states available for the full $N$-particle system, is given by the formula

$$
W_{N}=\frac{(G+(1-g)(N-1)) !}{N !(G-g N-(1-g)) !}
$$

where $G$ is the number of single-particle states. Clearly (5.6) and (5.7) reduces to standard expressions when $g=0$ (for bosons, with no exclusion) and $g=1$ (for fermions, with total exclusion). There exist some (theoretical) realizations of exclusion statistics for particles in one dimension (i.e. with two-dimensional phase space) for $g$ different from these two values. One particular case is the system of anyons confined to the lowest Landau level, which we have already considered [4]. In that case the exclusion statistics parameter $g$ is identical to the anyon statistics parameter $\nu$.

The statistical mechanics of particles with exclusion statistics can be de-

rived from the statistical weight (5.7) when the total energy can be written as a sum of single-particle energies and (5.7) is applied separately to (singleparticle) energy levels [21, 22, 23]. The result for the entropy is

$$
\begin{aligned}
S & =\sum_{k} D_{k}\left\{\left[1+(1-g) n_{k}\right] \ln \left[1+(1-g) n_{k}\right]\right. \\
& \left.+\left(1-g n_{k}\right) \ln \left(1-g n_{k}\right)-n_{k} \ln n_{k}\right\}
\end{aligned}
$$


where the sum runs over single-particle energy states. $D_{k}$ is the degeneracy of the $k$-th level and the quantum distribution function, $n_{k}$, is the average occupation number of the state $k$.

Since each quantum state occupies the phase space volume $h^{\mathcal{D}}$, with $2 \mathcal{D}$ the dimension of the single-particle phase space, we can relate $n$ and $\rho$ in the semiclassical limit by $n=\rho h^{\mathcal{D}}$. In the Boltzmann limit, $h \rightarrow 0$ and $n \rightarrow 0$, all dependence on $g$ in (5.8) goes away. If we, however, define the classical physics by the double limit $h \rightarrow 0, g \rightarrow \infty$ and $g h^{\mathcal{D}} \rightarrow \alpha$, where $\alpha$ is interpreted as a classical statistics parameter $\square$ (5.8) gets a nontivial limit of

$$
S=\sum_{k} D_{k} h^{\mathcal{D}}\left[\rho_{k} \ln \left(1-\alpha \rho_{k}\right)-\rho_{k} \ln \left(\rho_{k} h\right)+\rho_{k}\right] .
$$

If we further specialize to the case of fully degenerate states in a twodimensional phase space, where the sum is simply replaced by the total number of available single-particle states, $G=A / h$, and where $\rho_{k}$ is replaced by $N / A$, we exactly regain (5.4). This demonstrates that the classical statistical mechanics discussed in the previous section can be regarded as a special limit of exclusion statistics, different from the Boltzmann limit.

An alternative way to see the correspondence is to start from the the equation of state for exclusion statistics particles with the same energy,

$$
\beta P=\frac{G}{\mathcal{V}} \ln \left(1+\frac{n}{1-g n}\right),
$$

where $n=N / G$. Introducing the density $\tilde{\rho}=N / \mathcal{V}$ and taking the double limit defined above we get,

$$
\beta P=\frac{\tilde{\rho}}{1-\alpha \tilde{\rho} \frac{\mathcal{V}}{V_{1}}} .
$$

If we identify the the physical volume $\mathcal{V}$ with the one particle phase space volume $A$, so that $\tilde{\rho}=\rho$, we reproduce (5.5).

\footnotetext{
${ }^{11}$ Such a way of taking the classical limit is well-known from other contexts. Thus, a charged particle can in the quantum mechanical description be characterized by a dimensionless charge $g=q / \sqrt{\hbar c}$, where $q$ is the physical charge. (For $q=e$ we have $g^{2}=4 \pi \alpha$, with $\alpha$ the fine structure constant.) With $g$ fixed the charge $q$ depends on $\hbar$ and vanishes in the limit $\hbar \rightarrow 0$. However, if the classical limit is taken as $\hbar \rightarrow 0, g \rightarrow \infty$ with $g \sqrt{\hbar c} \rightarrow q$ the dimensional charge $q$ survives the classical limit.
} 
In the Appendix it is shown that even in a non-degenerate case, with particles in a harmonic oscillator potential, the classical statistical mechanics, defined as in Sect. 5A, coincides with that of exclusion statistics when the classical limit is taken in the way discussed above. Clearly what is important for the connection with exclusion statistics is the two defining relations (5.6) and (5.7) which determine the number of states in the system. In the classical description they are represented by the expressions for the phase

space volume, and by taking the limit $h \rightarrow 0, g h^{\mathcal{D}} \rightarrow \alpha$ it is straightforward to demonstrate that (5.6) and (5.7) reproduce the expressions for the phase space volume derived in Sects. 3 and 4.

\section{Discussion}

In this paper we have described a way to encode the particle statistics in the classical Lagrangian of a many particle system. The important point is that the Lagrangian includes more information about the system than just the classical equation of motion. It also gives information about the volume of the phase space, which in the quantum description corresponds to the number of states. If the $N$-particle volume can be determined as a function of the single particle volume, a classical statistics parameter can be defined as the reduction in available phase space volume for one particle by the presence of the others. Viewed in this way this classical statistics can be regarded as an analogue of exclusion statistics. In the specific examples we have considered, this relation can be made more specific and the classical statistical mechanics derived from this can be seen as a special way to take classical limit of exclusion statistics.

To make this idea more precise we have considered cases where the classical mechanics can be derived from the quantum description by constraining the motion in Hilbert space to (generalized) coherent states. For bosons, fermions and even anyons with a two-dimensional phase space the Lagrangian can be derived and the phase space volume can be calculated. The dimensional, classical statistics parameter, defined as the volume occupied by each particle present, in these cases are simply the dimensionless quantum statistics parameter multiplied with Planck's constant $h$. In another example, vortices in CSGL theory, there is no such underlying point particle description, but a similar classical Lagrangian can be found and the classical statistics parameter can be related to the coupling of the Chern-Simons term. 
There are several interesting questions raised by this description:

- Is the "classical fermion" description useful in some cases? This description would correspond to retaining the fermions' ability to occupy phase space, but otherwise treat them as classical particles. (A classical electron would then be characterized both by a charge and a (classical) statistics parameter.) Can the description give a useful approximation for other objects, like vortices in superfluids or superconductors?

- In the examples we have studied the phase space is two-dimensional, but the formalism (like for exclusion statistics) does not seem to depend in any crucial way on dimension. Are there non-trivial higher-dimensional examples? (Fermions in two and three dimensions can certainly be represented like this.)

- What about quantizing such a classical theory? In the cases we have studied, with a Kähler metric defined on phase space, a quantum description can presumably be derived in a unique way by use of analyticity properties. When regarded as a "re-quantization" of the system, how does it relate to the original quantum description. What would in particular the quantum description of the CSGL vortices be?

All these questions seem to merit further investigation.

\section{Appendix: Harmonic oscillator potential}

In this Appendix we consider the statistical mechanics of particles in a harmonic oscillator potential. The particles are "classical anyons" in the sense discussed in Sect. 3, i.e. the one derived from quantum anyons in the lowest Landau level. The system can also be interpreted as a coherent state representation of particles in a one-dimension harmonic oscillator potential, in a form interpolating between bosons and fermions. We calculate the partition function of the $N$-particle system and show that this is related to the partition function of a (quantum) system of particles with exclusion statistics in a harmonic oscillator potential by the same correspondence as obtained in Sect. 4.

The wave functions of the lowest Landau level have the form

$$
\psi(\mathbf{z}, \overline{\mathbf{z}})=\prod_{i<j}\left(\bar{z}_{i}-\bar{z}_{j}\right)^{\nu} f(\overline{\mathbf{z}}) e^{-\frac{1}{2} \overline{\mathbf{z}} \mathbf{z}},
$$

with $f(\overline{\mathbf{z}}) \equiv f\left(z_{1}, \ldots, z_{N}\right)$ as a general anti-analytic function of the complex particle coordinates. It is assumed to be symmetric in the variables. We 
introduce analytic basis vectors by

$$
\langle\mathbf{z} \mid \psi\rangle=f(\overline{\mathbf{z}})
$$

The basis vectors $\left|z_{1}, \ldots, z_{N}\right\rangle$ are not normalized, but we assume $\nu$ to be chosen such that they are regular and non-vanishing at points of coincidence of particle positions. Normalized vectors are introduced by

$$
\begin{aligned}
\left|\psi_{\mathbf{z}, \overline{\mathbf{z}}}\right\rangle & =\mathcal{N}_{\mathbf{z}, \overline{\mathbf{z}}}|\mathbf{z}\rangle \\
\left|\mathcal{N}_{\mathbf{z}, \overline{\mathbf{z}}}\right|^{-2} & =\langle\mathbf{z} \mid \mathbf{z}\rangle
\end{aligned}
$$

Defined in this way $\left|\mathcal{N}_{\mathbf{z}, \mathbf{z}}\right|^{-2}$ is a regular function with no zeros anywhere in $N$-particle space, and the Kähler potential $K=\ln |\mathcal{N}|^{-2}$ is a regular function everywhere.

The Hamiltonian depends on two frequencies, the cyclotron frequency $\omega_{c}$ determined by the external magnetic field and the frequency $\omega_{0}$ of the additional harmonic oscillator potential. When acting on the anti-analytic part $f(\overline{\mathbf{z}})$ of the wave functions of LLL, the Hamiltonian has the form

$$
\begin{aligned}
H & =\hbar\left(\omega_{t}-\omega_{c}\right) \sum_{i} \bar{z}_{i} \partial_{\bar{z}_{i}}+\hbar \omega_{t}\left[\frac{\nu}{2} N(N-1)+\frac{N}{2}\right] \\
& =\hbar \omega \sum_{i} \bar{z}_{i} \partial_{\bar{z}_{i}}+V_{N}^{0}
\end{aligned}
$$

with $\omega_{t}=\sqrt{\omega_{c}^{2}+\omega_{0}^{2}}, \omega=\omega_{t}-\omega_{c}$ and $V_{N}^{0}$ the quantum mechanical ground state energy

$$
V_{N}^{0}=\hbar \omega_{t}\left[\frac{\nu}{2} N(N-1)+\frac{N}{2}\right]
$$

For a system of particles in a one-dimensional harmonic oscillator potential the Hamiltonian is essentially the same, except that it depends on a single frequency $\omega_{0}$,

$$
H=\hbar \omega_{0} \sum_{i} \bar{z}_{i} \partial_{\bar{z}_{i}}+\hbar \omega_{0}\left[\frac{\nu}{2} N(N-1)+\frac{N}{2}\right] .
$$

Thus the difference between these two cases is only an overall $N$-dependent shift of the energy spectrum. 
The energy of the classical description is determined by the matrix elements of the Hamiltonian (A.4),

$$
\begin{aligned}
V(\mathbf{z}) & =\langle\mathbf{z}|H| \mathbf{z}\rangle\left|\mathcal{N}_{\mathbf{z}, \overline{\mathbf{z}}}\right|^{2} \\
& =\left[\left\{\hbar \omega \sum_{i} \bar{z}_{i} \partial_{\bar{z}_{i}}+\hbar \omega_{t}\left[\frac{\nu}{2} N(N-1)+\frac{N}{2}\right]\right\}\left|\mathcal{N}_{\mathbf{z}, \overline{\mathbf{z}}}\right|^{-2}\right]\left|\mathcal{N}_{\mathbf{z}, \overline{\mathbf{z}}}\right|^{2} \\
& =\hbar \omega \sum_{i} \bar{z}_{i} \partial_{\bar{z}_{i}} \ln \left|\mathcal{N}_{\mathbf{z}, \overline{\mathbf{z}}}\right|^{-2}+\hbar \omega_{t}\left[\frac{\nu}{2} N(N-1)+\frac{N}{2}\right]
\end{aligned}
$$

and the $N$-particle partition function is

$$
Z_{N}=\frac{1}{h^{N}} \int \frac{\omega^{N}}{N !} e^{-\beta V},
$$

where $\omega$ is the symplectic form

$$
\omega=-f_{\bar{z}_{i} z_{j}} d \bar{z}_{i} \wedge d z_{j}
$$

with

$$
f_{\bar{z}_{i} z_{j}}=i \hbar \partial_{\bar{z}_{i}} \partial_{z_{j}} \ln \left|\mathcal{N}_{\mathbf{z}, \overline{\mathbf{z}}}\right|^{-2}
$$

The form of the energy makes it possible to evaluate the integrals in the expression for the partition function. We write it as

$$
\begin{aligned}
Z_{N}=\frac{e^{-\beta V_{N}^{0}}}{\pi^{N} N !} \epsilon_{i j \ldots k} \int d^{2} z_{1} \ldots d^{2} z_{N} & {\left[\partial_{\bar{z}_{i}} \partial_{z_{1}} \ln \left|\mathcal{N}_{\mathbf{z}, \overline{\mathbf{z}}}\right|^{-2} \ldots \partial_{\bar{z}_{k}} \partial_{z_{N}} \ln \left|\mathcal{N}_{\mathbf{z}, \overline{\mathbf{z}}}\right|^{-2}\right] } \\
& \times \exp \left\{-\beta\left[\hbar \omega \sum_{i} z_{i} \partial_{z_{i}} \ln \left|\mathcal{N}_{\mathbf{z}, \overline{\mathbf{z}}}\right|^{-2}\right]\right\}
\end{aligned}
$$

The partition function can be rewritten as

$$
\begin{aligned}
Z_{N}= & \frac{1}{(-\beta \hbar \omega)} \frac{e^{-\beta V_{N}^{0}}}{\pi^{N} N !} \epsilon_{i j \ldots k} \int d^{2} z_{1} \ldots d^{2} z_{N} \frac{1}{z_{1}} \partial_{\bar{z}_{i}}\left[\ln \left|\mathcal{N}_{\mathbf{z}, \overline{\mathbf{z}}}\right|^{-2} \ldots\right. \\
& \left.\times \partial_{\bar{z}_{k}} \partial_{z_{N}} \ln \left|\mathcal{N}_{\mathbf{z}, \overline{\mathbf{z}}}\right|^{-2} \exp \left\{-\beta\left(\hbar \omega \sum_{i} z_{i} \partial_{z_{i}} \ln \left|\mathcal{N}_{\mathbf{z}, \overline{\mathbf{z}}}\right|^{-2}\right)\right\}\right]
\end{aligned}
$$


and by use of the identity

$$
\frac{1}{z_{1}} \partial_{\bar{z}_{i}}=\partial_{\bar{z}_{i}} \frac{1}{z_{1}}-\pi \delta\left(z_{1}\right) \delta_{i 1}
$$

the integration over $z_{1}$ can be performed

$$
\begin{aligned}
Z_{N} & =\frac{\pi}{\beta \hbar \omega} \frac{e^{-\beta V_{N}^{0}}}{\pi^{N} N !} \epsilon_{j \ldots k} \int d^{2} z_{2} \ldots d^{2} z_{N}\left[\partial_{\bar{z}_{j}} \partial_{z_{2}} \ln \left|\mathcal{N}_{\mathbf{z}, \overline{\mathbf{z}}}\right|^{-2} \ldots\right. \\
& \left.\times \partial_{\bar{z}_{k}} \partial_{z_{N}} \ln \left|\mathcal{N}_{\mathbf{z}, \overline{\mathbf{z}}}\right|^{-2}\right] \exp \left\{-\beta\left[\omega \sum_{i} z_{i} \partial_{z_{i}} \ln \left|\mathcal{N}_{\mathbf{z}, \overline{\mathbf{z}}}\right|^{-2}\right]\right\}
\end{aligned}
$$

The $(N-1)$-particle integral in this expression is of the same form as the original $N$-particle integral, and by repeating the procedure $N$ times we get the following simple expression for the partition function

$$
\begin{aligned}
Z_{N} & =\frac{1}{(\beta \hbar \omega)^{N} N !} e^{-\beta V_{N}^{0}} \\
& =\frac{1}{(\beta \hbar \omega)^{N} N !} \exp \left\{-\beta \hbar \omega_{t}\left[\frac{\nu}{2} N(N-1)+\frac{N}{2}\right]\right\} .
\end{aligned}
$$

The classical expression for the partition function can be compared with the partition function of the quantum system

$$
Z_{N}=\operatorname{Tr} e^{-\beta H},
$$

with $H$ given by (A.4). This expression is easily evaluated, since it can be written as

$$
\begin{aligned}
Z_{N} & =e^{-\beta V_{N}^{0}} \sum_{l_{1}=0}^{\infty} \sum_{l_{2}=l_{1}}^{\infty} \ldots \sum_{l_{N}=l_{N-1}}^{\infty} e^{-\beta \hbar \omega \sum_{i} l_{i}} \\
& =e^{-\beta V_{N}^{0}}\left[\prod_{n=1}^{N}\left(1-e^{-n \hbar \beta \omega}\right]^{-1} .\right.
\end{aligned}
$$

This expression shows that in the limit $\hbar \rightarrow 0$, with $\hbar \nu$ fixed, the partition function (A.18) of the quantum system coincides with the classical partition function (A.15). (Note however that the classical function depends on $\hbar$ explicitly, not only through the statistics factor $\alpha=h \nu$, due to the contribution from the ground state energy.) 
It is well known that the system of particles in the lowest Landau level can be regarded as a special realization of exclusion statistics [4], and the correspondence between the two partition functions discussed here is therefore essentially the same as the correspondence between the classical statistical mechanics and the statistical mechanics of particles with exclusion statistics discussed in Sect. 4. If we use the harmonic oscillator as a volume regulator the relation between the discussion in this Appendix and in Sect. 4 becomes even more direct. The thermodynamic limit is here taken by interpreting the limit $\omega_{0} \rightarrow 0$ in a specific way [24]. For the quantum case the harmonic oscillator regulator has been used in [22], and the expressions for the entropy and equation of state of anyons in the LLL were found in this way. Due to the correspondence between the quantum and classical descriptions, the thermodynamic limit of the classical functions with the harmonic oscillator regularization, will be identical to the corresponding functions of Sect. 4 . That is what should be expected, since for the thermodynamic limit it should be of no significance whether volume regularization is done by confinement to a sphere or by confinement in a harmonic oscillator potential.

\section{Acknowledgment}

Serguei Isakov acknowledges the support received through a NATO Science Fellowship granted by the Norwegian Research Council. S.I. also appreciates warm hospitality of NORDITA during his stay there in the summer of 1999, where part of this work was done.

Three of us (T.H.H., S.I. and J.M.L.) would also like to thank the Department of physics, Norwegian University of Science and Technology (Trondheim) for the inspiring atmosphere of the Workshop on low-dimensional physics in June 1999. 


\section{References}

[1] M.G.C. Laidlaw and C.M.DeWitt, Feynman functional integrals for systems of indistinguishable particles, Phys. Rev. D 3 (1971) 1375.

[2] J.M. Leinaas and J. Myrheim, On the Theory of Identical Particles, Nuovo Cimento 37B (1977) 1.

[3] G.A. Goldin, R. Menikoff and D.H. Sharp, Particle Statistics from Induced Representations of a Local Current Group, J. Math. Phys. 21 (1980) 650 ;

Representations of a Local Current Algebra in Nonsimply Connected Space and the Aharonov-Bohm Effect, J. Math. Phys. 22 (1981) 1664; F. Wilczek, Magnetic flux, angular momentum and statistics, Phys. Rev. Lett. 48 (1982) 1144 ;

Quantum mechanics of fractional-statistics particles, Phys. Rev. Lett. 49 (1982) 957.

[4] F.D.M. Haldane, Fractional statistics in arbitrary dimensions - A generalization of the Pauli principle, Phys. Rev. Lett. 67 (1991) 937.

[5] T.M. Samols, Vortex Scattering, Commun. Math. Phys. 145 (1992) 149.

[6] N.S. Manton, Statistical mechanics of vortices, Nucl. Phys. B 400 [FS] (1993) 624.

[7] M.V. Berry, Quantal phase factors acompanying adiabatic changes, Proc. Roy. Soc. (London) A 392 (1984) 45.

[8] R. Jackiw and L. Faddeev Hamiltonian reduction of unconstrained and constrained systems, Phys. Rev. Lett. 60 (1988) 1692.

[9] J.P. Provost and G. Vallee, Riemannian Structure on Manifolds of Quantum States, Commun. Math. Phys. 76 (1980) 289.

[10] A. Perelemov, Generalized Coherent States and Their Applications Springer-Verlag 1986.

[11] See e.g., A. Karlhede and E. Westerberg, Anyons in a magnetic field, Int. Jour. Mod. Phys. B 6 (1992) 1595. 
[12] H. Kjønsberg and J.M. Leinaas, On the anyon description of the Laughlin hole states, Int. Jour. Mod. Phys. A 12 (1997) 1975.

[13] H. Kjønsberg and J. Myrheim, Numerical study of charge and statistics of Laughlin quasi particles, Int. Jour. Mod. Phys. A 14 (1999) 537.

[14] F. D. M. Haldane, Fractional Quantization of the Hall Effect: A Hierarchy of Incompressible Quantum Fluid States, Phys. Rev. Lett. 51 (1983) 605 .

[15] F.D.M. Haldane and E.H. Rezayi, Periodic Laughlin-Jastrow wave functions for the fractional quantized Hall effect, Phys. Rev. B 31 (1985) 2529 .

[16] N. S. Manton, S. M. Nasir, Volume of Vortex Moduli Spaces (partition functions on a Riemann surface), Commun. Math. Phys. 199 (1999) 591.

[17] S. M, Girvin and A. H. MacDonald, Off-Diagonal Long-Range Order, Oblique Confinement, and the Fractional Quantum Hall Effect. Phys. Rev. Lett. 58, 1252 (1987); S. C. Zhang, T. H. Hansson and S. A. Kivelson, Effective-field-theory model for the fractional quantum Hall effect, Phys. Rev. Lett. 62 (1989) 82.

[18] N. S. Manton, "First order vortex dynamics," Annals Phys. 256, 114 (1997) hep-th/9701027.

[19] C.H. Taubes, Arbitrary N-Vortex Solutions to the First Order GinzburgLandau Equations, Commun. Math. Phys. 72 (1980) 277, 75 (1980) 207.

[20] D.Arovas, R.Schriffer and F.Wilczek, Fractional Statistics and the Quantum Hall Effect, Phys. Rev. Lett. 53 (1984) 722

[21] S.B. Isakov, Generalization of statistics for several species of identical particles, Mod. Phys. Lett. B 8 (1994) 319;

Fractional statistics in one dimension: modeling by means of $1 / x^{2}$ interaction and statistical mechanics; Int. J. Mod. Phys. A 9 (1994) 2563.

[22] A. Dasnières de Veigy and S. Ouvry, Equation of state of an anyon gas in a strong magnetic field, Phys. Rev. Lett. 72 (1994) 600. 
[23] Y.S. Wu, Statistical distribution for generalized ideal-gas of fractionalstatistics particles, Phys. Rev. Lett. 73 (1994) 922.

[24] A. Comtet, Y. Georgelin and S. Ouvry, Statistical aspects of the anyon model, J. Phys. A: Math. Gen. 22 (1989) 3917; K. Olaussen, On the harmonic oscillator regularization of partition function, Trondheim Univ. preprint No. 13 (1992), cond-mat/9207005. 